\title{
DUSTY TIME FRACTIONAL MHD FLOW OF A NEWTONIAN FLUID THROUGH A CYLINDRICAL TUBE WITH A NON-DARCIAN POROUS MEDIUM
}

\author{
H. Imo Mani Singha, Sanjib Sengupta \\ Department of Mathematics, Assam University \\ Silchar, India \\ imomanisingha@gmail.com,sanjib_aus2009@rediffmail.com
}

Received: 3 May 2020; Accepted: 3 December 2020

\begin{abstract}
In this paper, time fractional flow of a Newtonian fluid through a uniform cylindrical tube with a non-Darcy porous medium in the presence of dust particles under the application of a uniform magnetic field along the meridian axis is discussed. The implication of time fractional order differential equations in flow problems and some benefits of fractional order differential equations are highlighted. The Laplace Decomposition Method (LDM) is used to obtain an approximate solution to the proposed problem. The impact of fractional order and integer order of the differential equations and also the effects of some important parameters on the flow system are shown in the forms of graphs and a table. The convergence test of the solution is done. It has been observed that the fractional order differential equation reveals more things like the decrease in dust particle velocity due to the increase in magnetic field for fractional order derivatives, whereas, no noticeable change in dust particle velocity due to the change in magnetic field for integer order derivatives are observed. Also, it is observed that an increase in a fractional order derivative decrease the fluid as well as the dust particle velocities. The skin friction at the walls of the tube are also highlighted.
\end{abstract}

MSC 2010: 35Q30, 76D05, 35C10, 35E15, 35R11, 76S05, 76W05, 35A25, 44A10, 76T99, 49M27, 65M55

Keywords: time-fractional order Navier-Stokes equation, Laplace decomposition method (LDM), magnetohydrodynamics (MHD), dusty flow, non-Darcy porous medium

\section{Introduction}

The study of fluid flow, especially the biomagnetic fluid in the presence of dust particles or impurities is of utmost importance in the present day. It can be widely found in biomedical sciences including blood flow like the behaviour of blood flow in the presence of a magnetic field. In the last few decades, fractional Navier-Stokes equations have played a very important role in dealing with such problems. There are several methods to solve fractional Navier-Stokes equations. But out of all those methods, the Laplace decomposition method (LDM) is one of the best methods. 
Jafari et al. [1] used the LDM to solve linear and nonlinear fractional diffusion wave equations. Kumar et al. [2] found the analytical solution of fractional Navier-Stokes equations by the modified LDM. Ali et al. [3] used the LDM to obtain the analytical solution of the general Fisher's equation. Yousef et al. [4] used the LDM to solve the delay differential equations with an initial value problem. Mahmood et al. [5] used the LDM to deal with the multidimensional model of time fractional Navier-Stokes equations.

Our study is to detect the behaviour of the biomagnetic fluid flow in the presence of dust particles and a uniform magnetic field through a uniform cylindrical tube with porous medium. In recent years, many works have been developed on the study of the flow of the biomagnetic fluid like blood inside the body. Eldesoky [6] studied slip effects on the unsteady MHD pulsatile blood flow through a porous medium in an artery under the effect of body acceleration. Kumar et al. [7] modeled blood flow in an inclined tapered artery under the MHD effect through a porous medium. Shah et al. [8] also studied the effects of the fractional order and magnetic field on the blood flow in cylindrical domains. Topoliceanu et al. [9] gave a study on the blood two-phase dusty fluid flow. Attia [10] studied the magnetohydrodynamics (MHD) flow of a dusty fluid through a circular pipe considering the Hall effect. Gireesha et al. [11] studied the geometry of the unsteady motion of a dusty fluid through porous media in a uniform pipe. Attia et al. [12] studied the unsteady magnetohydrodynamics (MHD) flow and heat transfer of a dusty electrically conducting fluid between two infinite horizontal plates.

Motivated by the above works, this paper deals with the study of flow of a Newtonian biomagnetic fluid through a uniform cylindrical tube in the presence of a magnetic field. In addition, the fluid flow medium is considered to be a porous one. Furthermore, it is considered that the fluid contains impurities in the form of dust particles. A flow model for this situation in the form of the fractional Navier-Stokes equation is developed. Since it is not possible to obtain the general solution of the Navier-Stokes equation easily, certain assumptions are considered and an approximate solution of our fractional Navier-Stokes equation is obtained. In doing so, the Laplace Decomposition Method (LDM) is used to obtain an approximate solution. After successfully checking the convergence of the solution, graphs of the different parameters involved are obtained and a table is also highlighted. The main objective of this paper is to highlight the advantages of the time fractional order flow model over classical integer order flow models.

\subsection{Caputo Fractional Derivative}

Definition 1 A real function $f(\tau), \tau>0$ is said to be in the space $C_{\lambda}, \lambda \in \mathbb{R}$ if there exists a real number $d(>\lambda)$ such that $f(\tau)=\tau^{d} f_{1}(\tau)$, where $f_{1}(\tau) \in C[0, \infty)$ and is said to be in the space $C_{\lambda}^{n}$ iff $f^{(n)} \in C_{\lambda}, n \in \mathbb{N}$. 
Definition 2 The fractional derivative of a function $f(\tau)$ in Caputo sense is defined as

$$
D^{\beta} f(\tau)=\frac{1}{\Gamma(n-\beta)} \int_{0}^{\tau}(\tau-z)^{n-\beta-1} f^{(n)}(z) d z
$$

for $n-1<\beta \leq n, n \in \mathbb{N}, \tau>0, f \in C_{-1}^{n}$.

\subsection{Basic equations}

The basic equations for the flow of an incompressible fluid in a porous cylindrical domain $[13,14]$ are given by

$$
\begin{gathered}
\frac{D^{\alpha} \vec{q}}{D \tau^{\alpha}}=-\frac{1}{\rho} \nabla p+v \nabla^{2} \vec{q}-\frac{\vec{J} \times \vec{B}}{1-v_{p}} \vec{q}+\frac{\kappa N_{0}}{1-v_{p}}\left(\vec{q}-\vec{q}_{p}\right)-\frac{v}{\left(1-v_{p}\right) K} \vec{q}-\frac{c_{b}}{\left(1-v_{p}\right) \sqrt{K}} \vec{q}^{2} \\
m \frac{D^{\beta} \overrightarrow{q_{p}}}{D \tau^{\beta}}=\kappa\left(\vec{q}-\vec{q}_{p}\right)
\end{gathered}
$$

where $\vec{J}=\sigma(\vec{E}+\vec{q} \times \vec{B}), \vec{J}$ is current density, $\sigma$ is electrical conductivity, $\vec{E}$ is electric field, $\vec{B}$ is magnetic induction, $\vec{q}$ is the fluid velocity, $\vec{q}_{p}$ is the dust particle velocity, $v$ is the kinematic coefficient of viscosity, $\delta$ and $\eta$ are the particle mass parameter, $v_{p}$ is the volume fraction of the dust particle, $\rho$ is the density of the fluid particle, $p$ is the pressure, $\tau$ is the time, $\alpha$ and $\beta$ are time fractional derivatives, $K$ is the medium's permeability, $N_{0}$ is the number density of dust particle, $\kappa$ is the Stoke's resistance, $c_{b}$ is non-Darcian parameter and $\frac{D}{D \tau}=\frac{\partial}{\partial \tau}+q_{\psi} \frac{\partial}{\partial \psi}+\frac{q_{\theta}}{\psi} \frac{\partial}{\partial \theta}+q_{z} \frac{\partial}{\partial z}$. In our case, $\frac{\partial}{\partial \theta} \equiv 0$ (due to axi-symmetric flow) and also the velocity components along radial and meridian direction are also taken to be zero due to axial flow consideration i.e. if $\vec{q}=\left(q_{\psi}, q_{\theta}, q_{z}\right)$ then $q_{\psi}=q_{\theta}=0$ and thus, we have $\vec{q}=q_{z}(\psi, z, \tau)$. Also, we consider an infinite cylindrical tube which gives $\frac{\partial}{\partial z} \equiv 0$. Thus, (1) and (2) become

$$
\begin{gathered}
\frac{\partial^{\alpha} \vec{q}}{\partial \tau^{\alpha}}=-\frac{1}{\rho} \nabla p+v \nabla^{2} \vec{q}-\frac{\vec{J} \times \vec{B}}{1-v_{p}} \vec{q}+\frac{\kappa N_{0}}{1-v_{p}}\left(\vec{q}-\vec{q}_{p}\right)-\frac{v}{\left(1-v_{p}\right) K} \vec{q}-\frac{c_{b}}{\left(1-v_{p}\right) \sqrt{K}} \vec{q}^{2} \\
m \frac{\partial^{\beta} \vec{q}_{p}}{\partial \tau^{\beta}}=\kappa\left(\vec{q}-\vec{q}_{p}\right)
\end{gathered}
$$

The following are a few assumptions considered in this work: (i) The fluid under consideration is an incompressible Newtonian fluid, (ii) The flow is considered axisymmetric as well as axial in nature, (iii) Only the time fractional derivative of Navier- 
-Stokes equations is taken into consideration, (iv) The magnetic field is applied along the meridian axis i.e. for our case $\vec{B}=\left(0, B_{0}, 0\right)$ where $B_{0}$ is the component of the magnetic field in meridian axis, considered as uniform, (v) We consider the voltage difference along the rear ends of the tube to be very low and, as a result, the electric field is neglected i.e. for our case $\vec{E}=0$.

Definition 3 Let $g(\tau)$ be a function of $\tau$ specified for $\tau>0$. Then the Laplace transform of the function $g(\tau)$ is denoted and defined as [15]

$$
L\{g(\tau)\}=f(s)=\int_{0}^{\infty} e^{-s \tau} g(\tau) d \tau \text { where } s \in \mathbb{C}
$$

Definition 4 The Laplace transform of the Caputo fractional derivative is defined as [16]:

$$
L\left[D^{m \beta} u(y, \tau)\right]=s^{m \beta} L[u(y, \tau)]-\sum_{j=0}^{m-1} s^{m \beta-j-1} u^{(j)}(y, 0), m-1<m \alpha \leq m, m \in \mathbb{N}
$$

\section{Mathematical formulation of the problem}

The schematic representation of the flow system is shown in the following figure. We have considered a uniform cylindrical tube of radius $\psi$ with $z$-axis as the axis of the cylinder. We have also considered the medium through which the fluid passes to be a porous one, and the fluid which is allowed to pass contains dust particles, and a uniform magnetic field is also applied along the meridian axis. The volume fraction of the dust particles $v_{p}$ are also taken into consideration.

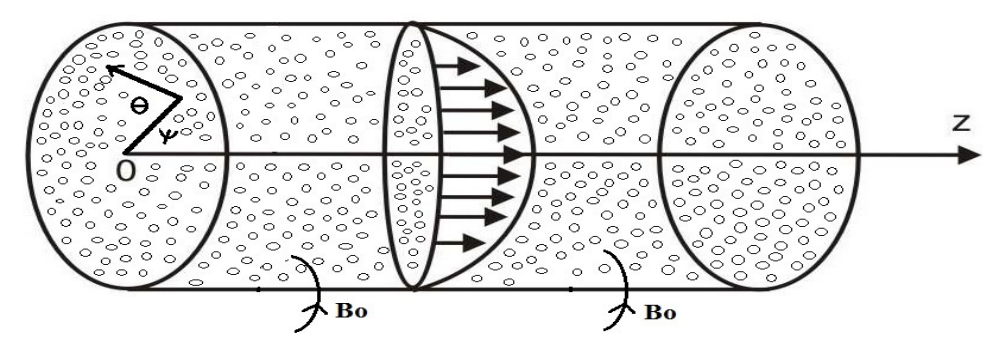

Fig. 1. Schematic diagram of the flow system

On the basis of the construction, we define a model of Newtonian fluid flow in a porous medium, with impure elements treated as dust particles present in it, through a uniform cylindrical tube in the presence of an applied magnetic field. We consider the flow to be taking place in the axial direction of the tube and the magnetic field 
being applied perpendicularly to the direction of the flow. Therefore, taking into consideration the assumptions made, we get our proposed model from (3) and (4) as

$$
\begin{array}{r}
\rho\left(1-v_{p}\right) \frac{\partial U^{*}}{\partial \tau^{*}}=\left(1-v_{p}\right)\left[-\frac{\partial p^{*}}{\partial z^{*}}+\frac{\mu}{\psi^{*}} \frac{\partial}{\partial \psi^{*}}\left(\psi^{*} \frac{\partial U^{*}}{\partial \psi^{*}}\right)\right]-\sigma B_{0}^{2} U^{*}-\kappa N_{0}\left(U^{*}-U_{p}^{*}\right) \\
-\frac{v}{K^{*}} U^{*}-\frac{c_{b}}{\sqrt{K^{*}}} U^{* 2}
\end{array}
$$

$$
m N_{0} \frac{\partial U_{p}^{*}}{\partial \tau^{*}}=\kappa N_{0}\left(U^{*}-U_{p}^{*}\right)
$$

We define the following non-dimensional quantities as

$$
\psi=\frac{\psi^{*}}{\psi_{0}}, U=\frac{U^{*}}{U_{\psi}}, U_{p}=\frac{U_{p}^{*}}{U_{\psi}}, \tau=\frac{\tau^{*}}{\tau_{\psi}}, p=\frac{p^{*} t_{\psi}}{\rho U_{\psi}}, z=\frac{z^{*}}{\psi_{0}} .
$$

where $\psi_{0}$ is the reference radius, $U_{\psi}$ is the reference velocity, $\tau_{\psi}$ is the reference time.

Thus, after non-dimensionalising equations (6) and (7), and also considering the pressure gradient to be constant throughout the flow, we write our proposed model in time-fractional derivative form as

$\frac{\partial^{\alpha} U}{\partial \tau^{\alpha}}=P+\frac{1}{R e}\left(\frac{\partial^{2} U}{\partial \psi^{2}}+\frac{1}{\psi} \frac{\partial U}{\partial \psi}\right)-\frac{H_{a}^{2}}{1-v_{p}} U-\frac{\delta \eta}{1-v_{p}}\left(U-U_{p}\right)-\frac{1}{\left(1-v_{p}\right) K} U-\frac{F r}{1-v_{p}} U^{2}$

$$
\frac{\partial^{\beta} U_{p}}{\partial \tau^{\beta}}=\eta\left(U-U_{p}\right)
$$

where $\alpha, \beta \in(0,1], U$ is the fluid velocity vector, $U_{p}$ is the dust particle velocity vector, $P$ is the constant pressure gradient, $\tau$ is the time, $\psi$ is the radius of the tube, $v_{p}$ is the volume fraction of the dust particle, $\alpha$ and $\beta$ are the time-fractional order parameters, $R e$ is the Reynold's number, $H_{a}^{2}$ is the Square of Hartmann number, $\delta$ and $\eta$ are the particle mass parameters, $F r$ is the Forchheimer coeffcient of the porous medium and $K$ is permeability of the medium.

\section{Laplace Decomposition Method (LDM)}

The LDM is widely used to get a solution of a non-linear differential equation $[1,2]$. Let us consider the general nonlinear fractional partial differential equation:

$D^{m \beta} U(y, \tau)+R[y] U(y, \tau)+N[y] U(y, \tau)=g(y, \tau), \quad \tau>0, y \in \mathbb{R}, m-1<m \alpha \leq m$ 
where $D^{m \beta}=\frac{\partial^{m \beta}}{\partial \tau^{m \beta}}, m \in \mathbb{N}$ and $R[y], N[y]$ denote the linear and nonlinear terms in $y$ and $g(y, \tau)$ are continuous functions. Next, we apply the Laplace transform on both sides of (10) and get

$$
\begin{array}{r}
L\left[D^{m \beta} U(y, \tau)\right]+L[R[y] U(y, \tau)+N[y] U(y, \tau)]=L[g(y, \tau)], \quad \tau>0, y \in \mathbb{R}, \\
m-1<m \alpha \leq m
\end{array}
$$

And then applying inverse Laplace transform on (11), we get

$$
U(y, \tau)=g(y, \tau)-L^{-1}\left[s^{-m \beta} L[R[y] U(y, \tau)+N[y] U(y, \tau)]\right]
$$

where $g(y, \tau)$ represents the term arising from the source term and the prescribed initial conditions.

The Laplace decomposition method assumes a series solution of the form

$$
U(y, \tau)=\sum_{n=0}^{\infty} U_{n}(y, \tau)
$$

The nonlinear term $N[y] U(y, \tau)$ is decomposed as [17]

$$
N[y] U(y, \tau)=\sum_{n=0}^{\infty} A_{n}
$$

where $A_{n}$ are called Adomian polynomials of $U_{0}, U_{1}, U_{2}, \ldots, U_{n}$, and it is defined as

$$
A_{n}=\frac{1}{n !} \frac{d^{n}}{d \tau^{n}}\left[N\left(\sum_{k=0}^{\infty} \lambda^{k} U_{n}\right)\right]_{\lambda=0}, n=0,1,2,3, \ldots
$$

Now, using all these in (12), we introduce the recursive relation as

$$
\begin{aligned}
& U_{0}(y, \tau)=g(y, \tau) \\
& \quad U_{n+1}(y, \tau)=-L^{-1}\left[s^{-m \beta} L\left[R[y] U_{n}+A_{n}\right]\right], n \geq 0
\end{aligned}
$$

Theorem 1 Let $N$ be an operator from a Hilbert space $H$ into $H$ and $U$ be the exact solution of (10). $\sum_{n=0}^{\infty} U_{n}$ which is obtained by (16) converges to $U$ if there exists $0 \leq$ $\alpha<1$ such that $\left\|U_{k+1}\right\| \leq \alpha\left\|U_{k}\right\| \forall k \in \mathbb{N} \cup\{0\}$ where $\|$.$\| is the supremum norm$ [18].

Definition 5 For every $n \in \mathbb{N} \cup\{0\}$, we define [18]

$$
\alpha_{n}=\left\{\begin{array}{c}
\frac{\left\|U_{n+1}\right\|}{\left\|U_{n}\right\|},\left\|U_{n}\right\| \neq 0 \\
0,\left\|U_{n}\right\|=0
\end{array}\right.
$$




\section{Solution of the problem by LDM}

Case I. With initial conditions $U(\psi, 0)=a\left(1-\psi^{2}\right)=U_{p}(\psi, 0)$ where $\psi \in[0,1]$ and $a>0$, the LDM is used to obtain the solutions of (8) and (9).

Using the Laplace decomposition method on (8), we get

$$
\begin{gathered}
U_{0}(\psi, \tau)=a\left(1-\psi^{2}\right)+P \frac{\tau^{\alpha}}{\Gamma(\alpha+1)} \\
U_{1}(\psi, \tau)=\xi_{1} \frac{\tau^{\alpha}}{\Gamma(\alpha+1)}+\xi_{2} \frac{\tau^{2 \alpha}}{\Gamma(2 \alpha+1)}+\xi_{3} \frac{\tau^{3 \alpha}}{\Gamma(3 \alpha+1)} \\
U_{2}(\psi, \tau)=\xi_{4} \frac{\tau^{2 \alpha}}{\Gamma(2 \alpha+1)}+\xi_{5} \frac{\tau^{3 \alpha}}{\Gamma(3 \alpha+1)}+\xi_{6} \frac{\tau^{4 \alpha}}{\Gamma(4 \alpha+1)} \\
-\frac{F r}{1-v_{p}} \cdot \frac{2 P \xi_{2}}{\Gamma(\alpha+1) \Gamma(2 \alpha+1)} \Gamma(4 \alpha+1) \cdot \frac{\tau^{5 \alpha}}{\Gamma(5 \alpha+1)}+\frac{\delta \eta^{2} P}{1-v_{p}} \cdot \frac{\tau^{2 \alpha+\beta}}{\Gamma(2 \alpha+\beta+1)}
\end{gathered}
$$

and so on. Thus, we get $\alpha_{0}=0.556783<1, \alpha_{1}=0.725141<1$ and so on. And using Theorem (1), we can conclude that our series solution obtained by the LDM is convergent.

Again, with the similar operation as above, we get the following from (9)

$$
\begin{gathered}
U_{p_{0}}=a\left(1-\psi^{2}\right) \\
U_{p_{1}}=\eta P \frac{\tau^{\alpha+\beta}}{\Gamma(\alpha+\beta+1)} \\
U_{p_{2}}=\eta \xi_{1} \frac{\tau^{\alpha+\beta}}{\Gamma(\alpha+\beta+1)}+\eta \xi_{2} \frac{\tau^{2 \alpha+\beta}}{\Gamma(2 \alpha+\beta+1)}+\eta \xi_{3} \frac{\tau^{3 \alpha+\beta}}{\Gamma(3 \alpha+\beta+1)} \\
-\eta^{2} P \frac{\tau^{\alpha+2 \beta}}{\Gamma(\alpha+2 \beta+1)}
\end{gathered}
$$

and so on. Thus, we get $\alpha_{0}^{\prime}=0.75<1, \alpha_{1}^{\prime}=0.710445<1$ and so on as above. And using Theorem (1), we can conclude that our series solution obtained by the LDM is convergent.

Case II. With initial conditions $U(\psi, 0)=a \psi=U_{p}(\psi, 0)$ where $\psi \in[0,1]$ and $a>0$, the LDM is used to obtain the solutions of (8) and (9).

Using the Laplace decomposition method on (8), we get

$$
\begin{aligned}
U_{0} & =a \psi+P \frac{\tau^{\alpha}}{\Gamma(\alpha+1)} \\
U_{1} & =\xi_{7} \frac{\tau^{\alpha}}{\Gamma(\alpha+1)}+\xi_{8} \frac{\tau^{2 \alpha}}{\Gamma(2 \alpha+1)}+\xi_{9} \frac{\tau^{3 \alpha}}{\Gamma(3 \alpha+1)}
\end{aligned}
$$




$$
\begin{array}{r}
U_{2}=\xi_{10} \frac{\tau^{2 \alpha}}{\Gamma(2 \alpha+1)}+\xi_{11} \frac{\tau^{3 \alpha}}{\Gamma(3 \alpha+1)}+\xi_{12} \frac{\tau^{4 \alpha}}{\Gamma(4 \alpha+1)}-\frac{F r \Gamma(4 \alpha+1)}{1-v_{p}} . \\
\frac{2 P \xi_{9}}{\Gamma(\alpha+1) \Gamma(3 \alpha+1)} \cdot \frac{\tau^{5 \alpha}}{\Gamma(5 \alpha+1)}+\frac{\delta \eta^{2} P}{1-v_{p}} \cdot \frac{\tau^{2 \alpha+\beta}}{\Gamma(2 \alpha+\beta+1)}
\end{array}
$$

and so on. Thus, we get $\alpha_{0}^{\prime \prime}=0.549767<1, \alpha_{1}^{\prime \prime}=0.713351<1$ and so on as above. And using Theorem (1), we can conclude that our series solution is convergent.

Again by using the Laplace decomposition method, we get the following from (9)

$$
\begin{aligned}
& U_{p_{0}}=a \psi \\
& U_{p_{1}}=\eta P \frac{\tau^{\alpha+\beta}}{\Gamma(\alpha+\beta+1)} \\
& U_{p_{2}}=\eta \xi_{7} \frac{\tau^{\alpha+\beta}}{\Gamma(\alpha+\beta+1)}+\eta \xi_{8} \frac{\tau^{2 \alpha+\beta}}{\Gamma(2 \alpha+\beta+1)}+\eta \xi_{9} \frac{\tau^{3 \alpha+\beta}}{\Gamma(3 \alpha+\beta+1)} \\
&-\eta^{2} P \frac{\tau^{\alpha+2 \beta}}{\Gamma(\alpha+2 \beta+1)}
\end{aligned}
$$

and so on. Thus, we get $\alpha_{0}^{\prime \prime \prime}=0.75<1, \alpha_{1}^{\prime \prime \prime}=0.709645<1$ and so on as above. And also Theorem (1), we can conclude that our series solution is convergent. The unknown terms are highlighted in the Appendix section at the end of this paper.

\section{Results and discussion}

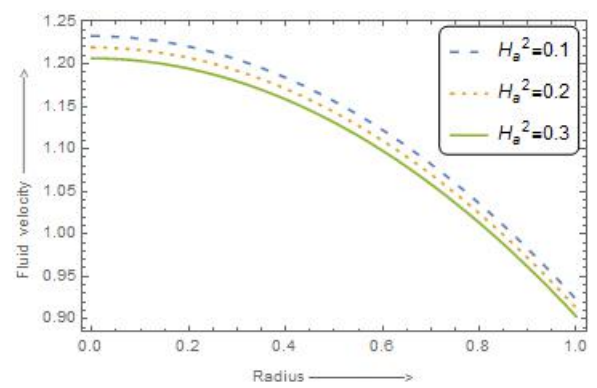

(a) $U$ vs $\psi\left(H_{a}^{2}\right.$ varies $)$

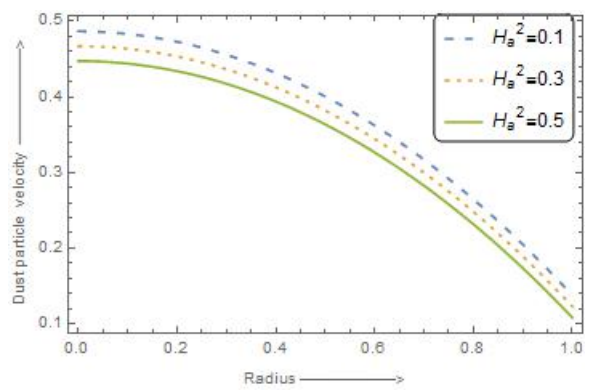

(b) $U_{p}$ vs $\psi\left(H_{a}^{2}\right.$ varies $)$

Fig. 2. $U$ vs $\psi$ and $U_{p}$ vs $\psi$ ( $H_{a}^{2}$ varies $)$ in Case-I

The values of parameters used throughout are $v_{p}=0.03, P=5, \delta=0.5, \eta=0.6$, $a=0.4, K=0.9, F r=0.5, \tau=0.1$. In Figures 2 (with fixed parameters $R e=$ $=500, \alpha=0.7, \beta=0.5$ ) the parametric influence of the magnetic field parameter placed along the meridian axis direction decreases the fluid as well as the dust particle velocities as it increases. This is due to the application of a magnetic field to the flow in a normal direction. A Lorentz force is produced which acts against the flow resulting in the decrease of fluid velocity. Figures 3 (with fixed parameters $R e=500$, 


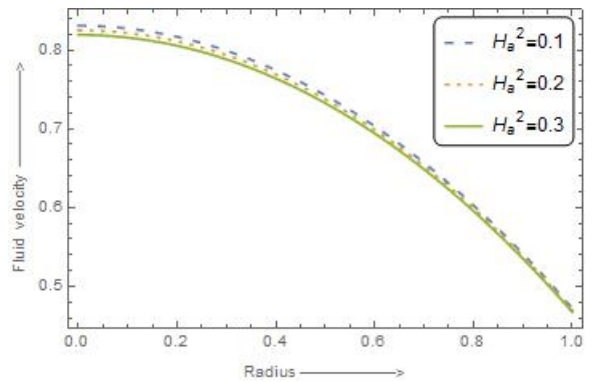

(a) $U$ vs $\psi\left(H_{a}^{2}\right.$ varies with $\left.\alpha=\beta=1\right)$

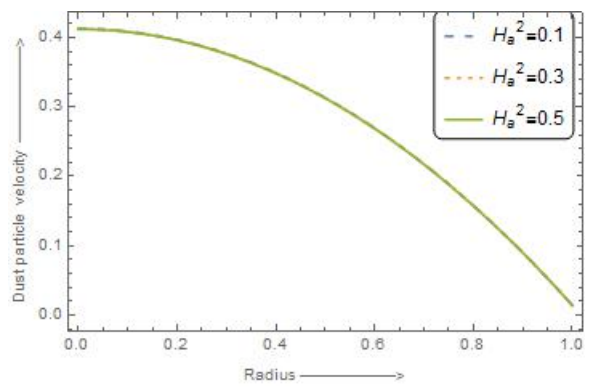

(b) $U_{p}$ vs $\psi\left(H_{a}^{2}\right.$ varies with $\left.\alpha=\beta=1\right)$

Fig. 3. $U$ vs $\psi$ and $U_{p}$ vs $\psi\left(H_{a}^{2}\right.$ varies with $\left.\alpha=\beta=1\right)$ in Case-I

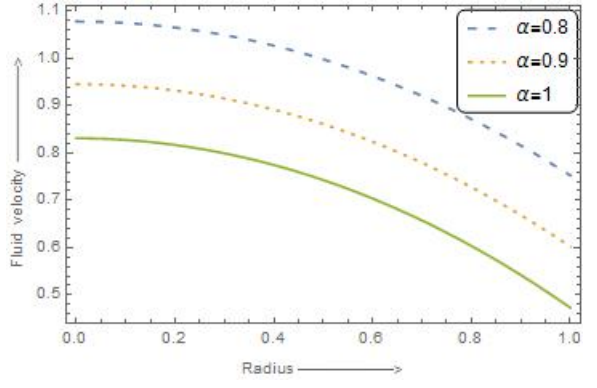

(a) $U$ vs $\psi(\alpha$ varies $)$

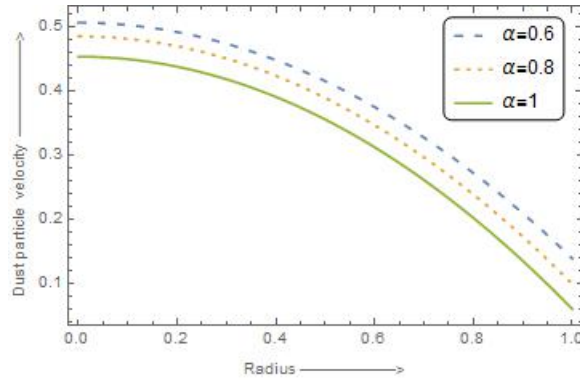

(b) $U_{p}$ vs $\psi(\alpha$ varies $)$

Fig. 4. $U$ vs $\psi$ and $U_{p}$ vs $\psi$ ( $\alpha$ varies) in Case-I

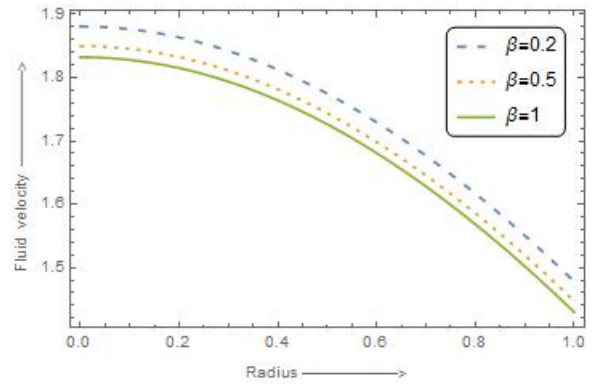

(a) $U$ vs $\psi(\beta$ varies $)$



(b) $U_{p}$ vs $\psi(\beta$ varies $)$

Fig. 5. $U$ vs $\psi$ and $U_{p}$ vs $\psi$ ( $\beta$ varies) in Case-I

$\alpha=1, \beta=1$ ) show that the change in the magnetic field does not show any noticeable change in the dust particle velocity if we take the time fractional order parameters $\alpha=\beta=1$. Figures 4 (with $R e=500, H_{a}^{2}=0.1, \beta=0.5$ ) highlight that the increment in time fractional order $\alpha$ decreases the fluid as well as the dust particle velocities. A similar pattern is observed for the time fractional order $\beta$ in Figures 5 (with $R e=500, H_{a}^{2}=0.1, \alpha=0.5$ ). In Figures 6 (with $R e=10, \alpha=0.8, \beta=0.5$ ), the parametric influence of the magnetic field parameter placed along the meridian 


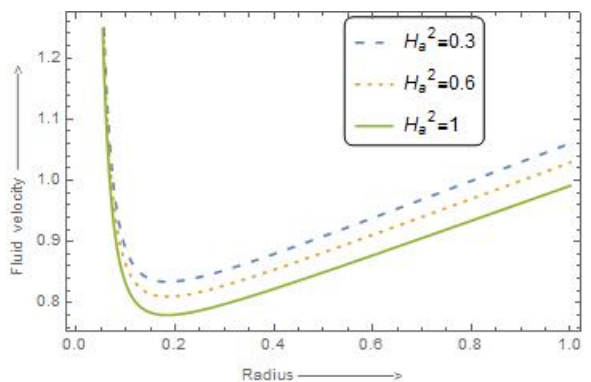

(a) $U$ vs $\psi\left(H_{a}^{2}\right.$ varies $)$

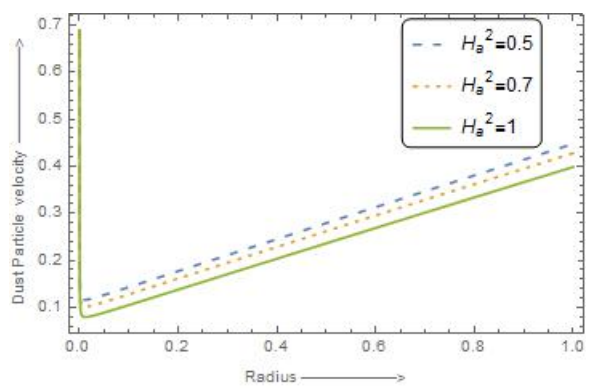

(b) $U_{p}$ vs $\psi\left(H_{a}^{2}\right.$ varies $)$

Fig. 6. $U$ vs $\psi$ and $U_{p}$ vs $\psi\left(H_{a}^{2}\right.$ varies $)$ in Case-II

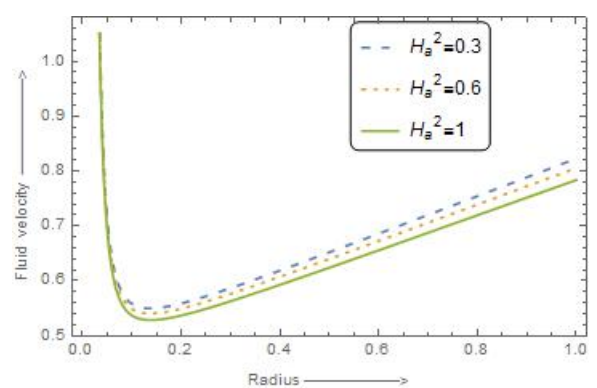

(a) $U$ vs $\psi\left(H_{a}^{2}\right.$ varies with $\left.\alpha=\beta=1\right)$

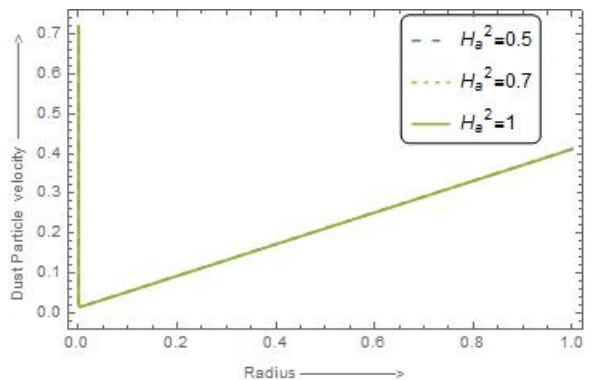

(b) $U_{p}$ vs $\psi\left(H_{a}^{2}\right.$ varies with $\left.\alpha=\beta=1\right)$

Fig. 7. $U$ vs $\psi$ and $U_{p}$ vs $\psi\left(H_{a}^{2}\right.$ varies with $\left.\alpha=\beta=1\right)$ in Case-II

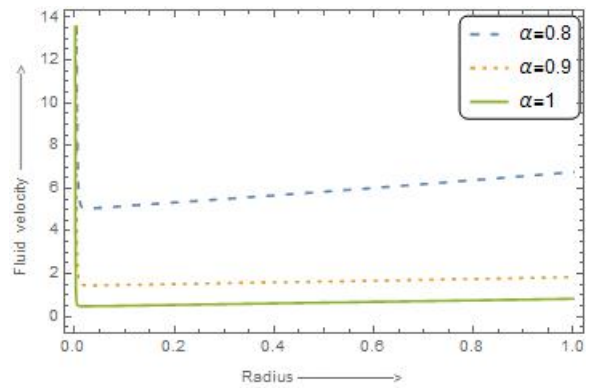

(a) $U$ vs $\psi$ ( $\alpha$ varies)

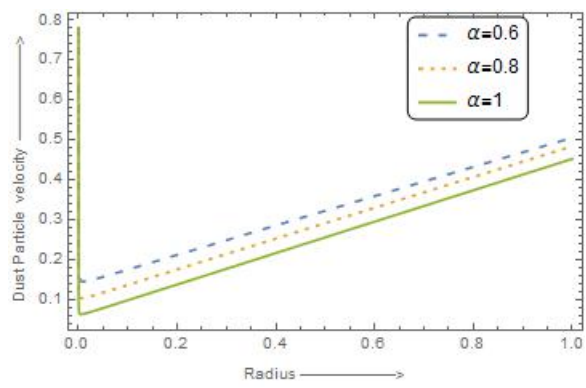

(b) $U_{p}$ vs $\psi(\alpha$ varies $)$

Fig. 8. $U$ vs $\psi$ and $U_{p}$ vs $\psi$ ( $\alpha$ varies) in Case-II

axis direction to the flow is found to decrease the fluid velocity and the dust particle velocity as it increases because of the induced Lorentz force. In Figures 7 (with $R e=10, \alpha=1, \beta=1$ ), we see that the change in the magnetic field does not show any noticeable change in the dust particle velocity if we take the time fractional order parameters $\alpha=\beta=1$. In Figures 8 (with $R e=500, H_{a}^{2}=0.1, \beta=0.5$ ), the fluid as well as the dust particle velocity decrease with the rise in the value of $\alpha$. In Figures 9 (with $R e=900, H_{a}^{2}=0.1, \alpha=0.5$ ), we observe the same pattern for $\beta$ as in the case 


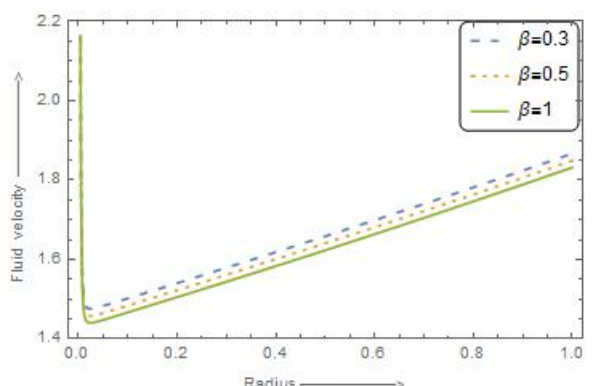

(a) $U$ vs $\psi(\beta$ varies $)$

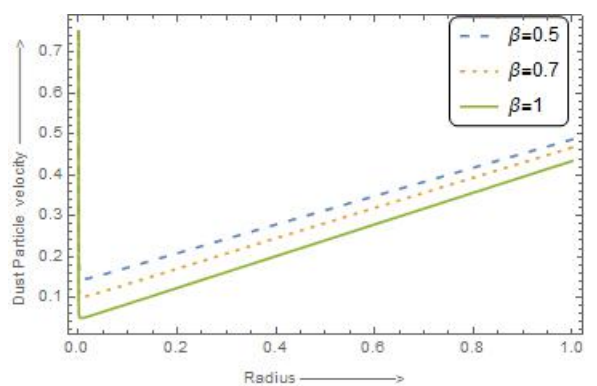

(b) $U_{p}$ vs $\psi(\beta$ varies $)$

Fig. 9. $U$ vs $\psi$ and $U_{p}$ vs $\psi$ ( $\beta$ varies) in Case-II

Table 1. Skin friction for Case-I and Case-II

\begin{tabular}{|c|c|c|c|c|c|c|}
\hline$H_{a}^{2}$ & $\alpha$ & $\beta$ & $F r$ & $R e$ & Skin friction in Case-I & Skin friction in Case-II \\
\hline $\mathbf{0 . 1}$ & 0.7 & 0.5 & 0.5 & 500 & 0.630332 & -0.305116 \\
\hline $\mathbf{0 . 2}$ & 0.7 & 0.5 & 0.5 & 500 & 0.62227 & -0.302725 \\
\hline $\mathbf{0 . 3}$ & 0.7 & 0.5 & 0.5 & 500 & 0.614752 & -0.300607 \\
\hline 0.1 & $\mathbf{0 . 8}$ & 0.5 & 0.5 & 500 & 0.667319 & -0.31813 \\
\hline 0.1 & $\mathbf{0 . 9}$ & 0.5 & 0.5 & 500 & 0.703051 & -0.33597 \\
\hline 0.1 & $\mathbf{1}$ & 0.5 & 0.5 & 500 & 0.730655 & -0.351746 \\
\hline 0.1 & 0.5 & $\mathbf{0 . 7}$ & 0.5 & 500 & 0.753524 & -0.432531 \\
\hline 0.1 & 0.5 & $\mathbf{0 . 8}$ & 0.5 & 500 & 0.753524 & -0.432531 \\
\hline 0.1 & 0.5 & $\mathbf{0 . 9}$ & 0.5 & 500 & 0.753524 & -0.432531 \\
\hline 0.1 & 0.5 & 0.6 & $\mathbf{0 . 6}$ & 500 & 0.826661 & -0.498621 \\
\hline 0.1 & 0.5 & 0.6 & $\mathbf{0 . 7}$ & 500 & 0.917964 & -0.579908 \\
\hline 0.1 & 0.5 & 0.6 & $\mathbf{0 . 8}$ & 500 & 1.02743 & -0.67639 \\
\hline 0.1 & 0.5 & 0.6 & 0.5 & $\mathbf{1 0 0}$ & 0.756691 & -0.432801 \\
\hline 0.1 & 0.5 & 0.6 & 0.5 & $\mathbf{2 0 0}$ & 0.754712 & -0.432634 \\
\hline 0.1 & 0.5 & 0.6 & 0.5 & $\mathbf{3 0 0}$ & 0.754052 & -0.432577 \\
\hline
\end{tabular}

of $\alpha$. Table 1 represents the skin friction $=-\left(\frac{\partial U}{\partial \psi}\right)_{\psi=1}$ at the walls of the tube for both Case-I and Case-II. Here, it is observed that due to an increase in $H_{a}^{2}$ and $R e$, the magnitude of the values of skin friction decreases. Also, due to increasing values of $\alpha$ and $F r$, the magnitude of the values of skin friction increases. It is also interesting to find that the change in values of $\beta$, the magnitude of the skin friction does not change, which indicates that the skin friction is not affected by the presence of dust particles in the flow domain. 


\section{Conclusion}

A theoretical study of a time-fractional incompressible Newtonian viscous fluid is considered through a non-Darcy porous medium in a uniform cylindrical tube with dust particle immersion under the influence of a uniform magnetic field along the meridian axis. The Laplace Decomposition Method (LDM) is used to find an approximate solution to our proposed time fractional order differential equation. It is observed that the presence and increase of magnetic field parameter $H_{a}^{2}$, timefractional parameter $\alpha, \beta$ reduces the velocity flow rate of fluid as well as the dust particle flow rate. It has been observed that a noticeable change in dust particle velocities occur only when we take non-integer values of time fractional orders $\alpha$ and $\beta$. But if we take $\alpha=\beta=1$, then no noticeable change is observed in the dust particle velocity. Thus, the time fractional orders play a very important role in the fractional order fluid flow. It is observed that the graphs that showed no noticeable change for integer order derivatives are found to show a noticeable change and variation for fractional order derivatives. Therefore, we can conclude that taking fractional order time derivatives has its own benefits in studying a fluid flow system which can be of much importance in many fields of sciences. The present work can be extended to study the behaviour of flow of non-Newtonian fluids like that of blood flow in human body under certain conditions.

\section{Appendix}

- $\xi_{1}=-\frac{4 a}{R e}-\left(\frac{H_{a}^{2}}{1-v_{p}}+\frac{1}{K\left(1-v_{p}\right)}\right) a\left(1-\psi^{2}\right)-\frac{F r}{1-v_{p}} a^{2}\left(1-\psi^{2}\right)^{2}$

- $\xi_{2}=-\left(\frac{H_{a}^{2}}{1-v_{p}}+\frac{1}{K\left(1-v_{p}\right)}+\frac{\delta \eta}{1-v_{p}}+\left(\frac{F r}{1-v_{p}} 2 a\left(1-\psi^{2}\right)\right)\right) P$

- $\xi_{3}=\frac{-F r \cdot P^{2}}{1-v_{p}} \cdot \frac{\Gamma(2 \alpha+1)}{(\Gamma(\alpha+1))^{2}}$

- $\xi_{4}=\frac{4 a}{R e}\left(\frac{H_{a}^{2}}{1-v_{p}}+\frac{1}{K\left(1-v_{p}\right)}\right)-\frac{F r \cdot a^{2}}{\operatorname{Re}\left(1-v_{p}\right)}\left(16 \psi^{2}-8\right)$

$-\left(\frac{H_{a}^{2}}{1-v_{p}}+\frac{1}{K\left(1-v_{p}\right)}+\frac{\delta \eta}{1-v_{p}}\right) \xi_{1}-\frac{F r}{1-v_{p}} \cdot \frac{2 a\left(1-\psi^{2}\right) \xi_{1}}{\Gamma(\alpha+1)} \Gamma(\alpha+1)$

- $\xi_{5}=\frac{8 a P F r}{\operatorname{Re}\left(1-v_{p}\right)}-\left(\frac{H_{a}^{2}}{1-v_{p}}+\frac{1}{K\left(1-v_{p}\right)}+\frac{\delta \eta}{1-v_{p}}\right) \xi_{2}$

$-\frac{F r}{1-v_{p}}\left\{\frac{2 a\left(1-\psi^{2}\right) \xi_{2}}{\Gamma(2 \alpha+1)}+\frac{2 P \xi_{1}}{(\Gamma(\alpha+1))^{2}}\right\} \Gamma(2 \alpha+1)$

- $\xi_{6}=-\left(\frac{H_{a}^{2}}{1-v_{p}}+\frac{1}{K\left(1-v_{p}\right)}+\frac{\delta \eta}{1-v_{p}}\right) \xi_{3}$

$-\frac{F r}{1-v_{p}}\left\{\frac{2 a\left(1-\psi^{2}\right) \xi_{3}}{\Gamma(3 \alpha+1)}+\frac{2 P \xi_{2}}{\Gamma(\alpha+1) \Gamma(2 \alpha+1)}\right\} \Gamma(3 \alpha+1)$ 
- $\xi_{7}=\frac{a}{\psi R e}-\left(\frac{H_{a}^{2}}{1-v_{p}}+\frac{1}{\left(1-v_{p}\right) K}\right) a \psi-\frac{F r}{1-v_{p}} a^{2} \psi^{2}$

- $\xi_{8}=-\left(\frac{H_{a}^{2}}{1-v_{p}}+\frac{1}{\left(1-v_{p}\right) K}\right) P-\frac{F r}{1-v_{p}} 2 a \psi P-\frac{\delta \eta}{1-v_{p}} P$

- $\xi_{9}=-\frac{F r}{1-v_{p}} \cdot \frac{P^{2}}{(\Gamma(\alpha+1))^{2}} \Gamma(2 \alpha+1)$

- $\xi_{10}=\frac{1}{R e}\left(\frac{a}{\psi^{3} R e}-\frac{4 a^{2} F r}{1-v_{p}}-\frac{a}{\psi}\left(\frac{H_{a}^{2}}{1-v_{p}}+\frac{1}{\left(1-v_{p}\right) K}\right)\right)$ $-\left(\frac{H_{a}^{2}}{1-v_{p}}+\frac{\delta \eta}{1-v_{p}}+\frac{1}{\left(1-v_{p}\right) K}\right) \xi_{7}-\frac{F r \Gamma(\alpha+1)}{1-v_{p}} \cdot \frac{2 a \psi \xi_{7}}{\Gamma(\alpha+1)}$

- $\xi_{11}=-\frac{2 a P F r}{\psi\left(1-v_{p}\right) R e}-\left(\frac{H_{a}^{2}}{1-v_{p}}+\frac{\delta \eta}{1-v_{p}}+\frac{1}{\left(1-v_{p}\right) K}\right) \xi_{8}$ $-\frac{F r \Gamma(2 \alpha+1)}{1-v_{p}}\left\{\frac{2 a \psi \xi_{8}}{\Gamma(2 \alpha+1)}+\frac{2 P \xi_{7}}{(\Gamma(\alpha+1))^{2}}\right\}$

- $\xi_{12}=-\left(\frac{H_{a}^{2}}{1-v_{p}}+\frac{\delta \eta}{1-v_{p}}+\frac{1}{\left(1-v_{p}\right) K}\right) \xi_{9}-$ $\frac{\operatorname{Fr} \Gamma(3 \alpha+1)}{1-v_{p}}\left\{\frac{2 a \psi \xi_{9}}{\Gamma(\alpha+1)}+\frac{2 P \xi_{8}}{\Gamma(\alpha+1) \Gamma(2 \alpha+1)}\right\}$

\section{References}

[1] Jafari, H., Khalique, C.M., \& Nazari, M. (2011). Application of the Laplace decomposition method for solving linear and nonlinear fractional diffusion wave equations. Applied Mathematics Letters, 24(11), 1799-1805.

[2] Kumar, S., Kumar, D., Abbasbandy, S., \& Rashidi, M. (2014). Analytical solution of fractional Navier-Stokes equation by using modifed Laplace decomposition method. Ain Shams Engineering Journal, 5(2), 569-574.

[3] Ali, A. \& Shah, K. (2018). Analytical solution of general Fisher's equation by using Laplace Adomian decomposition method. Journal of Pure and Applied Mathematics, 2(3), 01-04.

[4] Yousef, H.M. \& Ismail, A.M. (2018). Application of the Laplace Adomian decomposition method for solution system of delay differential equations with initial value problem. AIP Conference Proceedings, 1974(1), 020038.

[5] Mahmood, S., Shah, R., Arif, M. (2019). Laplace Adomian decomposition method for multi dimensional time fractional model of Navier-Stokes equation. Symmetry, 11(2), 149.

[6] Eldesoky, I.M. (2012). Slip effects on the unsteady MHD pulsatile blood flow through porous medium in an artery under the effect of body acceleration. International Journal of Mathematics and Mathematical Sciences, 2012.

[7] Kumar, A., Chandel, R., Shrivastava, R., Shrivastava, K., \& Kumar, S. (2016). Mathematical modeling of blood flow in an inclined tapered artery under mhd effect through porous medium. International Journal of Pure and Applied Mathematical Sciences, 9(1), 75-88.

[8] Shah, N.A., Vieru, D., \& Fetecau, C. (2016). Effects of the fractional order and magnetic field on the blood flow in cylindrical domains. Journal of Magnetism and Magnetic Materials, 409, 10-19. 
[9] Topoliceanu, F., Varvara, G., \& Zaharia, D. (1997). The blood two-phase dusty fluid flow modeling. IFAC Proceedings Volumes, 30(2), 39-44.

[10] Attia, H.A. (2011). Transient circular pipe mhd flow of a dusty fluid considering the hall effect. Kragujevac Journal of Science, 33, 15-23.

[11] Gireesha, B., Madhura, K., \& Bagewadi, C. (2012). Flow of an unsteady dusty fluid through porous media in a uniform pipe with sector of a circle as cross-section. International Journal of Pure and Applied Mathematics, 76(1), 29-47.

[12] Attia, H., Aboul-Hassan, A., Abdeen, M., \& Abdin, A.E.D. (2014). Mhd flow of a dusty fluid between two in finite parallel plates with temperature dependent physical properties under exponentially decaying pressure gradient. Bulgarian Chemical Communications, 46, 320-329.

[13] Shah, N.A., Vieru, D., \& Fetecau, C. (2016). Effects of the fractional order and magnetic field on the blood flow in cylindrical domains. Journal of Magnetism and Magnetic Materials, 409, 10-19.

[14] Hamid, M., Zubair, T., Usman, M., \& Haq, R.U. (2019) Numerical investigation of fractionalorder unsteady natural convective radiating flow of nanofluid in a vertical channel. AIMS Mathematics, 4(5), 1416-1429.

[15] Spiegel, M.R. (1965). Laplace Transforms. New York: McGraw-Hill.

[16] Podlubny, I. (1998). Fractional Differential Equations: An Introduction to Fractional Derivatives, Fractional Differential Equations, to Methods of Their Solution and Some of Their Applications (Vol. 198). Elsevier.

[17] Adomian, G. (1998). A review of the decomposition method in applied mathematics. Journal of Mathematical Analysis and Applications, 135(2), 501-544.

[18] Hosseini, M.M. \& Nasabzadeh, H. (2006). On the convergence of Adomian decomposition method. Applied Mathematics and Computation, 182(1), 536-543.

[19] Nagaraju, G., \& Garvandha, M., (2019). Magnetohydrodynamic viscous fluid flow and heat transfer in a circular pipe under an externally applied constant suction. Heliyon, e01281.

[20] Matta, A., \& Nagaraju, G., (2018). Order of chemical reaction and convective boundary condition effects on micropolar fluid flow over a stretching sheet. AIP Advances, 8, 115212.

[21] Ramana Murthy, J.V., \& Nagaraju, G., (2009). Flow of a couple stress fluid generated by a circular cylinder subjected to longitudinal and torsional oscillations. Contemporary Engineering Sciences, 2(10), 451-461.

[22] Nagaraju, G., \& Garvandha, M., (2020). Impacts of variable thermal conductivity and mixed convective stagnation-point flow in a couple stress nanofluid with viscous heating and heat source. Heat Transfer, 1-21.

[23] Ramana Murthy, J.V., Nagaraju, G., \& Sai, K.S., (2012). Numerical solution for MHD flow of micro polar fluid between two concentric rotating cylinders with porous lining. International Journal of Nonlinear Science, 13(2), 183-193.

[24] Nagaraju, G., Matta, A., \& Kaladhar, K., (2017). The effects of Soret and Dufour, chemical reaction, Hall and ion currents on magnetized micropolar flow through co-rotating cylinders. AIP Advances, 7, 115201.

[25] Nagaraju, G., \& Garvandha, M.,(2020). The influence of magnetized couple stress heat, and mass transfer flow in a stretching cylinder with convective boundary condition, cross-diffusion, and chemical reaction. Thermal Science and Engineering Progress, 18, 100517. 